\title{
L'aferesi tra arte e scienza
}

\author{
Erasmo Buongiorno
}

U.O.C. Nefrologia, Dialisi e Trapianto, Ospedale V. Fazzi, Lecce

\begin{abstract}
Apheresis between ART AND SCIENCE
Abstract. Art and science, emotion and reason, are the basic tools of knowledge and of the man's relationship with his surroundings, in a relationship sometimes harmonious, more often conflicting, but always fruitful. Today only experimental sciences seem to produce knowledge that is reproducible, verifiable, and able to explain the world as it is, thus relegating the human sciences to a secondary role. Therefore it becomes clear that a science separate from the philosophy of scientists cannot exist, because its directions and the realized contents are the outcome of complex cultural layers and lives in its time. Therapeutic apheresis is a borderland, where the technological applications are closely following the progress of science, but sometimes seem to precede it, creating their own way, dictating the timing of research, as a noble man's attempt to overcome his limitations.
\end{abstract}

Key words: Apheresis, Art, Science, Knowledge

Conflict of interest: None.

Financial support: None.

Ricevuto: 28 Gennaio 2013; Accettato: 28 Febbraio 2013

La creazione artistica è un'attività che riteniamo riservata esclusivamente agli esseri umani, perché atto di auto-espressione, ma quando, nel 1965, uno studente liceale di 17 anni, Raymond Kurzweil, il futuro ricercatore ed entusiasta divulgatore scientifico, ospite di uno show televisivo, I have Got a Secret, suonò al pianoforte un breve brano musicale, composto da un rudimentale computer da lui stesso costruito, la creatività umana apparve usurpata da una macchina e, così, cominciava ad affievolirsi il confine tra intelligenza biologica e intelligenza artificiale. E i computer, che diventano sempre più veloci con velocità sempre maggiore, potrebbero, in teoria, diventare capaci di qualcosa di sempre più vicino all'intelligenza umana. Se ciò accadesse, l'umanità (corpi, menti, civiltà) sarebbe completamente e irreversibilmente trasformata e si potrebbe prolungare la vita all'infinito, trasferendo le coscienze nei computer e vivendo al loro interno come software, per sempre (1). In questo caso, la tecnica avrebbe il sopravvento e la creazione artistica ma anche la stessa scienza potrebbero divenire superflue e, forse, i computer annienterebbero l'umanità.

Dopo questa agghiacciante incursione tra utopia e distopia, che, come icona, merita l'Urlo (Skrik) di E. Munch, torniamo al nostro tempo, che ora ci apparirà caldo e accogliente, e al nostro tema, Scienza e Arte, cultura scientifica e cultura umanistica, ragione e emozione-intuizione, forme e mezzi del comune e universale processo della conoscenza della nostra specie. Ma partiamo dalla mano, il logo scelto dagli organizzatori dell'VIII Convegno Nazionale di Aferesi Terapeutica.
Quasi 2 milioni di anni fa, strani primati di taglia media emergono dalle praterie africane e, in poche migliaia di anni, in varie ondate, colonizzano il pianeta; inizia il genere Homo che ha creato la Scienza e l'Arte. Dopo che l'uomo, che formerà la specie sapiens, si è separato dal genere Pan, all'interno della tribù degli Hominini, grazie all'acquisizione dell'andatura bipede completa e alla disponibilità dell'uso delle mani, queste hanno consentito l'attività prensile e il linguaggio dei segni, in parallelo con l'accrescimento del volume dell'encefalo e con l'evoluzione delle funzioni cognitive $(2,3)$. La Cueva de las Manos nella Patagonia argentina come le pitture rupestri di Altamira e di numerosi altri siti archeo-palentologici testimoniano la sacralità riposta dai nostri antenati nella meravigliosa capacità di articolazione e nella molteplicità delle funzioni della mano, che diviene metafora dell'“essere" e della consapevolezza di sé.

La mano è strumento essenziale della comunicazione gestuale e strumentale e consente il "manufatto" che, districandosi tra significante e significato, forma e contenuto, si muove verso la possibilità del pensiero astratto: la Venere di Willendorf (4), tra i primi manufatti pervenuti dotati di finalità solo estetiche risalente a 25-30.000 anni fa, diviene un'esaltazione della fertilità che descrive il canone estetico femminile e l'ideale di bellezza fisica legato alle condizioni socio-economiche e riconosciuto dalla società del tempo e che esprime consapevolezza della vita e della morte e la esorcizza, nella speranza della sopravvivenza della specie. 
La costante domanda "cosa c'è oltre l'orizzonte" che l'uomo si pone è allo stesso tempo oggetto e strumento di quell'agire e conoscere che vede mani e cervello in febbrile interconnessione bidirezionale costruire infaticabilmente il sapere dell'umanità, che è scienza e arte, ragione ed emozione.

Niccolò Stenone (Niels Stensen), il grande scienziato, medico e fondatore delle scienze della terra, luterano, poi vescovo e, poi, semplice prete missionario nei paesi riformati, morto in odore di santità e beatificato nel 1988, così mirabilmente riassumeva l'inesausto desiderio di conoscenza dell'umanità: "pulchra sunt quae videntur, pulchriora quae sciuntur, longe pulcherrima quae ignorantur" (5). Questo è straordinariamente evidente oggi che, con i moderni telescopi, guardando lontano nello spazio, guardiamo talmente indietro nel tempo da giungere a poter fotografare i primi momenti del big bang, quando nascono il tempo, la materia, lo spazio “... chiusi in una disperante finitezza, siamo, tuttavia, in grado di guardare nell'abisso dello spazio-tempo e siamo diventati testimoni dell'inizio degli inizi' (6).

Ma qual è il rapporto tra arte e scienza? È un'antinomia? Sono due caratteri in opposizione? Noi siamo abituati a pensare alla conoscenza scientifica come alla ricerca della verità, anche se provvisoria, certa finché non ne venga dimostrato l'errore (7), risultato di un processo cognitivo assolutamente razionale $\mathrm{e}$ contrapposta all' esperienza artistica basata, invece, sulla emozione e sull'intuizione, esito del prevalere delle componenti non razionali della persona. Comunemente siamo abituati a pensare nei termini hegeliani della dialettica tra tesi, antitesi e sintesi, resa popolare dalla divulgazione marxiana, come nel famoso sonetto di Francesco Petrarca, dove la prima quartina recita: "Pace non trovo, et non ò da far guerra; e temo, et spero; et ardo, et son un ghiaccio; et volo sopra 'l cielo, et giaccio in terra; et nulla stringo, et tutto 'l mondo abbraccio" (8).

Ma non è stato sempre così: nel grande affresco "La scuola di Atene" attribuito a Raffaello, le molteplici espressioni dell'intelletto umano, le sette arti liberali personificate nei grandi maestri del passato, sono in armonica relazione tra loro. È una polifonia governata da dialettica e retorica impersonate da Socrate e Aristotele al centro e poi grammatica e musica poste sotto la protezione di Apollo sul lato sinistro di chi guarda mentre, sulla destra, geometria, aritmetica e astronomia sono sotto la protezione di Minerva (9). Di questa armonia tra arte e scienza testimoniano grandi artisti dell'età dell'umanesimo e del Rinascimento, che erano anche studiosi di scienze della natura. Leonardo da Vinci, che è l'interprete straordinario dell'indissolubile legame tra scienza e arte, affermava che la pittura, che è comprensione della realtà fenomenica, ha una base scientifica, matematicamente misurabile, segno dell'armonia divina, "colta e condivisa dall'arte suprema del saper vedere".

A conferma di questo suo convincimento, Leonardo avrebbe applicato nella realizzazione delle sue grandi opere pittoriche le proporzioni dettate dalla sezione aurea, il cosiddetto linguaggio matematico della bellezza. Questa sezione del segmento $\mathrm{AB}$ nel punto $\mathrm{C}$ è tale che " $\mathrm{AB}: \mathrm{AC}=\mathrm{AC}: \mathrm{CB}$ ", dove entrambi $\mathrm{i}$ rapporti si approssimano a $\varphi \cong 1,618$. A questo rapporto che, per esempio, lega i due lati di una "prosaica" carta di credito il grande matematico fra' Luca Pacioli dedi- cò il libro De Divina Proportione, pubblicato a Venezia nel 1497, che ebbe grande influenza sugli artisti dell'epoca, da Leon Battista Alberti a Mantegna, e che recava illustrazioni attribuite a Leonardo.

Fino all'illuminismo, non ci sarà antitesi tra arte e scienza; è solo nell' 800 , con l'imporsi del positivismo, che celebra "dell'umana gente le magnifiche sorti e progressive", secondo l'amara ironia di Giacomo Leopardi (10), che si rompe l'armonia, perché il metodo scientifico diviene l'unica via per la conoscenza: "Keine Metaphisik mehr", niente più metafisica. Ma, tra la fine dell'800 e i primi anni del nuovo secolo, una serie di scoperte imbarazzanti mette in crisi queste certezze: da un lato la teoria dell'evoluzione e la scoperta dell'inconscio e, dall'altro, la meccanica quantistica e la teoria della relatività. Il solco tra arte e scienza si fa gradualmente sempre più profondo, né valgono i tentativi di ricomporre l'antinomia, come nella Vienna di quegli anni in cui sembra riannodarsi il dialogo progressivo tra arte e scienza con uno "scambio di intuizioni", di idee cruciali e di esperienze tra artisti modernisti nell'ambito delle arti figurative e della letteratura, musicisti, filosofi e medici, anche se emergevano modi nuovi di pensare il pensiero (11). Ed è in quegli anni che Sigmund Freud scrive ad Arthur Schnitzler, medico ma, soprattutto, grande interprete letterario della mitteleuropa: "Ho avuto l'impressione che Lei conosca attraverso l'intuizione, come attraverso dettagliate forme di osservazione, tutto ciò che io ho scoperto attraverso un faticoso lavoro sulle persone", dove arte medica e letteratura si tengono per mano.

Ma l'arte moderna, nel costante rifiuto della lezione del passato e delle sue tecniche rappresentative, sperimenta nuove identità e nuovi materiali per dire di una realtà scomposta, dove regnano il disordine e la disarmonia, dove l'attore della narrazione e della figurazione diventa moltitudine (Pessoa), uno, nessuno e centomila, (Pirandello), uomo senza qualità (Musil) e diventa le molte immagini di un caleidoscopio (Conrad) e le forme diffratte della pittura dal cubismo in poi. Ma, come in una jam session, ogni virtuosismo e ogni singolarità riportano a un sottostante ritmo comune, a una disperata ricerca di senso enfatizzata in un confronto muscolare, in una provocazione, in un continuo trascendere $\mathrm{i}$ confini della forma, pur oscillando tra iperrealismo, espressionismo e informale, quasi una disforia, tanto da far dire a Georges Braque "l'arte deve disturbare e la scienza deve rassicurare" o, come inaspettatamente suggerisce lo storico dell'arte, il viennese Ernst Gombrich: "La funzione biologica dell'arte è quella di sottoporci a una prova, di farci fare una ginnastica mentale che aumenti la nostra tolleranza a tutto ciò che non ci aspettiamo", anticipando il moderno legame tra arte e neuroscienze di cui Eric Kandel, laureato in discipline storico-letterarie, psichiatra e Nobel per gli studi sulla fisiologia della memoria, è straordinario interprete (10).

L'arte moderna però, forse perché desacralizzata nell'epoca della sua riproducibilità tecnica, privata di autenticità e unicità e dell" "aura" magica che le era propria (12) e banalizzata a intrattenimento e consolazione, cerca un riscatto nell'alterità e nell'estremizzazione, amplificando le distanze fino a farsi del tutto estranea al rigore del paradigma scientifico che rappresenta, per contro, un esito accettato e condiviso in attesa 
di nuovi modelli di riferimento, ma sempre interagente con la realtà sociale nel cui contesto si inserisce (13).

Nel panorama italiano la separazione tra scienza e arte è stata anche il prodotto di una durevole egemonia culturale dell'idealismo crociano, che vedeva la scienza (concetto) misuratrice della realtà e sottomessa alla filosofia (espressione), che permetterebbe la vera comprensione della realtà stessa. Scriveva, infatti, Benedetto Croce, nel 1902: "Il rapporto di conoscenza intuitiva o espressione e conoscenza intellettuale o concetto, di arte e scienza e di poesia e prosa non si può significare altrimenti se non dicendo ch'è quello di doppio grado. Il primo grado è l'espressione e il secondo è il concetto: il primo grado può stare senza il secondo, mentre il secondo non può stare senza il primo" (14). Questa disarmante concezione indignò Luigi Pirandello che, in uno smilzo libretto, "Arte e Scienza", del 1908, senza mezzi termini definiva il rapporto come "assolutamente arbitrario" "nell'aver fin da principio staccato con un taglio netto le varie attività e funzioni dello spirito".

Per Pirandello "Ogni opera di scienza è scienza e arte, come ogni opera d'arte è arte e scienza. Solo, come spontanea è l'arte nella scienza, così spontanea è la scienza nell'arte" (15). Alla stessa atmosfera culturale sembra appartenere l'esortazione di Friedrich Nietzsche a "guardare alla scienza con gli occhi dell'arte e all'arte con gli occhi della scienza", riportata da Paul Karl Feyerabend (16).

La sfida crociana verso la scienza positivista si concretizzerà in un'astiosa polemica contro il matematico Federigo Enriques ("il volenteroso Professor Enriques che, con zelo ma scarsa preparazione, si diletta di filosofia", dirà Croce), reo di aver organizzato il IV Congresso Internazionale di Filosofia a Bologna nell'Aprile del 1911, evento visto come luogo di convergenza di differenti saperi, come già accadeva nelle pagine di Scientia, la rivista da lui curata. La contesa era sulla possibilità che le scienze potessero essere strumento di conoscenza, come riteneva Enriques, e non mero strumento descrittivo, incapace di esprimere concetti, come sosteneva Croce.

La crescente distanza tra arte e scienza, tra cultura umanistica e cultura scientifica, pur concorrenti al comune universale processo della conoscenza, è stata avvertita da uomini di frontiera, come Charles P. Snow, fisico a Cambridge e scrittore, che, negli anni Cinquanta, si doleva del fatto che "Trent'anni fa le due culture non si rivolgevano la parola, ma almeno si sorridevano freddamente. Ora la cortesia è venuta meno e si fanno le boccacce" (17). In Italia, un convegno dal titolo "Due Culture?" a cura del Comitato Cattolico Docenti Universitari, nel Maggio del 1966 (18), ha testimoniato l'esigenza diffusa di comporre la distanza intercorsa tra due mondi una volta uniti, perché, come affermava W.E. Pauli, Nobel 1945 per la Fisica, "Tanto lo spirito umano che è in noi quanto l'oggetto percepito che è fuori di noi rientrano nello stesso ordine cosmico". Infine, in un bel dialogo a distanza, quasi un racconto epistolare, C. Bernardini fisico e T. De Mauro italianista si interrogano più recentemente sulla "denutrizione scientifica" e "sull'eccesso di pressappochismo" diventati piaghe nazionali (19).

Oggi, due aspetti antitetici sembrano proporsi, il primo figlio della critica anti-neo-positivista di P.K. Feyerabend, che tende a negare valore assoluto al metodo scientifico come modello di conoscenza, schierandosi dal lato delle avanguardie artistiche e della loro critica destrutturante, riconoscendo che la scienza procede per contaminazioni con altri saperi, tanto da non potersi dare una demarcazione, un'autonomia (20); il secondo modello emerge dai nichilismi del nostro tempo ed è ostaggio del dominio pervasivo della scienza e, soprattutto, della sua applicazione tecnica, capace di un inaudito controllo anche sulla sfera biologica, tanto da aver richiesto una fondazione e una rifondazione continua della bioetica (21). Certamente, solo le scienze sperimentali sembrano produrre conoscenze riproducibili e verificabili, in grado di spiegare il mondo com'è, mentre le scienze umane appaiono al massimo come "narrazioni e un modo di farsi coraggio, un fischiettare nel buio" (22).

E l'Aferesi? Utilizzando le categorie aristoteliche dell'agire dell'uomo, dobbiamo chiederci se essa attiene alla $\pi$ oteív, poiesis, l'agire tecnico e produttivo, dove l'azione, guidata dall'idea, è il mezzo per conseguire un fine, o se attiene alla $\pi \rho \alpha ́ \xi \eta$, praxis, l'agire pratico e morale, dove l'azione non è guidata dall'idea ma dall'ideale, è sostenuta da una disposizione interiore al bene e coincide con il fine stesso? A parer mio, in questa procedura che evoca una combinazione di arte, scienza sperimentale e tecnica applicativa, come la grande opera dei costruttori di cattedrali, nella sua forma produttiva è $\pi$ otcív, poiesis, ma, nella sua forma terapeutica, è $\pi \rho \alpha ́ \xi \eta$, praxis, cioè agire morale.

Perché, come in A. Camus "mi sono sentito all'improvviso un bisogno di impossibile. Le cose cosi come sono non mi sembrano soddisfacenti" (23), così, nel toccante racconto di A. Ramunni, entusiasta cultore della materia, nel quale l'aferesi si dimostra capace di restituire la vista a un amico, quando tutte le terapie note hanno fallito (24), si riconosce l'insopprimibile esigenza di non fermarsi a Itaca ma di cercare di raggiungere l'irraggiungibile orizzonte per guardare cosa c'è oltre: "siamo realisti, vogliamo l'impossibile" può esserne il motto.

E quando questa volontà è ispirata dalla ricerca del bene ( $\varphi \rho o ́ v \eta \sigma \eta \varsigma)$, allora la medicina si fa arte, perché esercitata con competenza tecnica e amore per l'umanità, tanto più se condotta senza il salvagente delle Linee Guida, fuori dalle rotte già battute, ma con spirito di pionieri. A tal riguardo, bene han fatto i due presidenti del convegno L. Gesualdo e A. Ramunni a rivolgere questa esortazione: "È opportuno che riacquistiamo la nostra creatività di Medici dediti alla battaglia contro le malattie, evitando le gabbie dei numeri e delle statistiche..." (25). Questo è tanto vero nell'Aferesi Terapeutica, in cui le applicazioni tecnologiche seguono da vicino l'avanzamento della scienza, ma talvolta sembrano precederla, sembrano cioè crearsi loro medesime la strada e dettare i tempi della ricerca, passo dopo passo, tanto più che l'incidenza/prevalenza delle patologie trattate, rare od orfane, non consentono spesso la realizzazione di trial. E, allora, mi viene in mente la citazione di Giuseppe Remuzzi sul fatto, cioè, che non esistano trial prospettici, randomizzati, controllati, in doppio cieco e crossover in grado di dimostrare l'utilità del paracadute per prevenire la morte e i traumi causati dai lanci dall'aereo (26), ma, nonostante ciò, il paracadute rimane un soddisfacente salvavita. Qui, devono intervenire, allora, la richiamata creatività, la peculiarità del rapporto antropologico medico-paziente che l'automatismo delle prestazioni protocollari, invece, mortifi- 
ca, perché la forza dei grandi numeri può attribuire al medico fragili certezze, pone al centro la malattia e non l'uomo ammalato e può consentire un risultato biologico, ma può lasciarsi sfuggire la salute dell'uomo osservato nella sua interezza e non come somma di organi e apparati.

$\mathrm{E}$, infine, l'aferesi come teoria e pratica segue il cammino della conoscenza, un cammino non lineare né progressivo, incontra sulla sua strada vicoli ciechi e sentieri che si biforcano fino a perdersi, esperimenta errori ed eventi casuali, anomie e ristrettezze, utilizza di volta in volta contenuti e modelli diversi, sperimentale e induttivo, congetturale e deduttivo e, ancora, talvolta, procede secondo la felice casualità; vive, dunque, la vita degli uomini e ne è irrinunciabile patrimonio.

“Non c'è un giorno da perdere: la ricerca va avanti...Qualcuno vuole raccogliere la sfida?” (27).

\section{Riassunto}

Arte e scienza, emozione e ragione rappresentano i fondamentali strumenti di conoscenza e di relazione dell'uomo con ciò che lo circonda, in un rapporto talora armonico, più spesso conflittuale, ma, sempre, fecondo. Oggi, quando solo le scienze sperimentali sembrano produrre conoscenze riproducibili, verificabili e in grado di spiegare il mondo com'è, relegando le scienze umane a un ruolo secondario, appare evidente, in realtà, come non possa esistere una scienza separata dalla filosofia di coloro che la coltivano, perché essa, sia nelle direzioni che intraprende che nei contenuti in cui si concretizza, è l'esito delle complesse stratificazioni culturali degli scienziati e vive nel suo tempo. L'Aferesi Terapeutica è una terra di confine, in cui le applicazioni tecnologiche seguono da vicino l'avanzamento della scienza, ma, talvolta, sembrano precederla, sembrano, cioè, crearsi loro stesse la strada e dettare i tempi della ricerca, in un nobile tentativo dell'uomo di superare la sua finitezza.

Parole chiave: Aferesi, Arte, Scienza, Conoscenza

Dichiarazione di conflitto di interessi: L'Autore dichiara di non avere conflitto di interessi.

Contributi economici degli autori: L'Autore dichiara di non aver ricevuto sponsorizzazioni economiche per la preparazione dell'articolo.

Indirizzo degli Autori:

Dr. Erasmo Buongiorno

U.O.C. Nefrologia, Dialisi e Trapianto

Ospedale V. Fazzi

Piazza F. Muratore 1

73100 Lecce

erasmo.buongiorno@libero.it

\section{Bibliografia}

1. Grossman L 2045. The Year Man Becomes Immortal. Time, Thursday, Feb. 10, 2011. http://www.time.com/time/magazine/ article/0,9171,2048299,00.html\#ixzz2Ip1f72Su.

2. Engels F. Parte avuta dal lavoro nel processo di umanizzazione della scimmia, 1876. Die Neue Zeitung 1896.

3. Cavalli Sforza LL, Pievani T. Homo Sapiens. La grande storia della diversità umana. Codice edizioni 2011.

4. Venere di Willendorf, Naturhistorisches Museum, Vienna.

5. Cutler A. La conchiglia del diluvio. Niccolò Stenone e la nascita della scienza della terra. Il Saggiatore Milano 2007.

6. Schiavone A. Storia e Destino. Einaudi 2007.

7. Popper K. Logica della ricerca scientifica. Il carattere autocorrettivo della scienza. Einaudi 1998.

8. Petrarca F. Canzoniere: sonetto n. 134 a cura di G. Contini. Einaudi 1964.

9. Glenn W. Most, Leggere Raffaello. La Scuola di Atene e il suo pre-testo. Einaudi 2001.

10. Leopardi G. Canti: La ginestra o il fiore del deserto. Einaudi 2005.

11. Kandel EK. L'età dell'inconscio. Arte, Mente e Cervello dalla grande Vienna ai nostri giorni. Raffaello Cortina 2012.

12. Benjamin W. L'opera d'arte nell'epoca della sua riproducibilità tecnica. Einaudi 2000.
13. Kuhn TS. La struttura delle rivoluzioni scientifiche. Einaudi 2009.

14. Croce B. Estetica come scienza dell'espressione e linguistica generale. Teoria e Storia. Adelphi 1990.

15. Pirandello L. Arte e scienza. Mondadori 1994.

16. Feyerabend PK. Contro l'autonomia. Il cammino delle scienze e delle arti. Mimesis 2012.

17. Snow CP. Le due culture. Marsilio 2005.

18. Due Culture? Atti del convegno di studio, Roma 20-21 Maggio 1966. Il Mulino 1966.

19. Bernardini C, De Mauro T. Contare e raccontare. Dialogo sulle due culture. Laterza 2005.

20. Feyerabend PK. Contro il metodo. Feltrinelli 1979.

21. Bellieni C. Rifondare la bioetica in Vita, Ragione, Dialogo. Scritti in onore di Elio Sgreggia. Cantagalli 2012.

22. Rosemberg A. The Atheist's Guide to Reality. WW Norton \& Company 2011.

23. Camus A. Caligola atto I, scena IV. Bompiani 2000.

24. Ramunni A. Una chiacchierata sull'aferesi terapeutica. Bios 2006.

25. Gesualdo L, Ramunni A. Introduzione all'VIII Congresso Nazionale di Aferesi terapeutica Bari 15-17 Novembre 2012.

26. Smith GC, Pell JP. Parachute use to prevent death and major trauma related to gravitational challenge: systematic review of randomised controlled trials. BMJ 2003; 327: 1459-61.

27. Remuzzi G. Arte e Ricerca 2003. Galleria Fumagalli 2003. 